\title{
The Use Of Physics In Answering Metaphysical Questions
}

Hoyt Edge, Ph.D.

Department of Philosophy, Rollins College

Because of the limits of my background, these comments will be restricted to the logic and structure of Janusz Slawinski's argument, as well as to the general philosophical context of the argument; I simply do not have the expertise to pass judgment on the adequacy of the biology and physics discussed in the paper. However, it turns out that the structure of the argument makes the material from science less important than it seems on first glance, because Slawinski never uses the material to argue for his theory.

Slawinski ought to be congratulated for his bold speculation and for the attempt to push forward the investigation of the survival question, surely one of the deepest, most intense, and persistent questions of Western culture. And yet, we must be clear about what he is actually doing in the paper, for appearances can be deceptive. In particular, it may appear that Slawinski is arguing for a specific theory of survival and is giving evidence from the forefront of science in support of that theory; but a closer examination of Slawinski's argument reveals that he does not do this. All that he really attempts to do is to show that his theory of survival is a possible theory, and in fact he gives virtually no support in favor of the theory, that is, why we ought to believe it. Let us take a closer look at two crucial junctures in his argument to see how this approach is played out.

In the section entitled "Necrotic Radiation and Its Interpretation," Slawinski asserts that there are three possible explanations of what happens in the death flash. The third is his own, and will not concern

Dr. Edge is Professor of Philosophy at Rollins College. Requests for reprints should be sent to Dr. Edge at the Department of Philosophy, Rollins College, Winter Park, FL 32789-4499. 
us here. The first possibility is that the death flash is a reflection of the entropy of the living system, and thus contains no real information. He asserts that he will give two arguments against this interpretation, by which I understand him to mean that he will show how this interpretation is inadequate. However, he does not do this; rather, he allows that the "conservation of $I$ is allowed within the framework of the second law of thermodynamics" (my emphasis). Further, using considerations from von Laue, he argues that the information content "might be preserved at the moment of death" (my emphasis). Neither "argument" gives us positive evidence for thinking that the first alternative to his own theory might be false; rather, they simply point to the possibility of this interpretation being inadequate, but we have no reason to think it is, in fact, insufficient.

Slawinski's argument against the second alternative is even more transparent, as he has to, in a rather odd ad hoc fashion, postulate a hidden force of nature behind the flash. Surely, such an argument presents no reason why we would want to doubt the adequacy of this interpretation.

I mentioned earlier that we must be clear about what Slawinski is doing. There are two points I want to make here. The first one, illustrated above, is that he is not really arguing for his theory. The second point is that his speculation deviates substantially from traditional speculation about minds and bodies, in which the two are thought to be of radically different natures. Now, my own view is that speculations undercutting this radical dualism are not only in order, but are probably correct; my objection, however, is that he has given us little direction in seeing how we can overcome that tradition.

For instance, in the section entitled "Low-Intensity Electromagnetic Radiation of Living Systems," he seems to identify mind with brain, a theory that has traditionally, except in idealism, served the function of reducing mind to brain, saying essentially that all mental qualities can be explained adequately in terms of physical theory. But there are conceptual problems with such a reduction that must be met and dealt with, if such a theory is going to be plausible. In that regard, it is inadequate to give an analogy of the ability of television to receive waves that picture the surface of the body, or even to extend it to holographic pictures, as such an analogy does not elucidate why we need to think of a person's intentions, motivations, feelings, ideals, loves, thoughts, and pains as being transportable in the same sort of way. Further, the analogy is not even clear, as the television waves certainly cannot be identified with the person's body; the picture on the screen is not the body itself. In the same sort of way, why would we 
want to say that the electromagnetic field is identified with the persons motivations, etc.? Such confusions abound in Slawinski's analysis. For instance, it is not at all clear how and why he can argue that embodied minds or auras are in space, but that after the death of the biological person, they are not in space, as they are not in time. How can something be so radically discontinuous and still be the same thing?

But then, the truth comes out: surviving consciousness is not all like embodied consciousness. Slawinski's theory requires the surviving consciousness to be a "seamless continuum," aware of and reacting to all things and all places. However interesting and logically possible that idea may be, it is certainly not obvious that either embodied consciousness or surviving consciousness is like that. Certainly, normal experience does not imply that embodied consciousness is such a continuum, and evidence from mediumistic communication suggests that surviving consciousness is neither terribly bright, nor knowledgeable, nor particularly interesting. Even the best mediumistic communication seems to be what the philosopher C. D. Broad called "a blend of twaddle and uplift." Again, I am not arguing that either embodied or surviving consciousness cannot be such a seamless continuum, only that one needs to present arguments showing its plausibility, which Slawinski does not do.

My comments are not aimed at showing the impossibility of Slawinski's speculation; quite the contrary, we ought to let a thousand theories bloom. But his speculation cuts across traditional thinking so much that we have a right to expect more convincing arguments than we have received. I simply do not find the bold claim that consciousness is nothing but an electromagnetic field to be either helpful or plausible, based upon an assertion that itself has no positive arguments presented in its favor, but only the reassurance that, however much the theory does not seem plausible from the view of contemporary science, it nevertheless does not conflict with science. In short, as far as I can tell, we have not received one argument from science or one argument from metaphysics for the theory.

I cannot leave my comments, however, without reacting to the terribly interesting point that Slawinski makes about time. When he connects his theory of electromagnetic consciousness with relativity theory, he postulates that surviving consciousness is sent out from the body at the speed of light. That has interesting consequences, one of which is that this electromagnetic field has "jumped" outside of the space/time continuum and therefore is not "in time." Slawinski points out that survival does not mean "life everlasting;" that is, survival should not be thought of in terms of continuing to exist forever. 
Although this point has been made by others, $I$ think it is valuable to make it again, and using the analogy from physics is helpful. But I think that it is an analogy only. I fear that Slawinski still is working with a literal definition of time, one that can be mathematically formalized. For instance, continuing to take time literally within the formalism of relativity theory, one could point out that embodied consciousness could "survive," in that if an embodied person were to travel close to the speed of light, he or she would have virtual continued existence, which would simulate survival for all practical purposes. But something is missing here when we treat survival purely in terms of mathematical formalisms, and the problem is not simply the distinction between (a) existing in a virtually continuous fashion "in time" on the one hand, and (b) going the speed of light and thus "flipping" out of space/time. The problem is rather handling survival literally, as a category subsumable under mathematical formalisms. There are so many other experiences of time, such as biological rhythms, subjective time, and psychological time, in which the notion of survival may be mined for insight, that we ought to take Slawinski's argument here more as an indication of the varied possibilities in grasping the notion of survival than as a literal description of survival.

Indeed, my major objection to Slawinski is his attempt to take what is a profound religious and metaphysical idea and to emasculate it by making it formal and literal; but that is what is bound to happen if one does not take seriously enough what is happening in contemporary science. At least since the seventeenth century, science has conceived of itself as providing literal truth, believing that the world can be described in only one way, and that the scientific method is the only legitimate source of that one truth. If one remains in that tradition and simply plays with unusual concepts developed in the forefront of science, one retains the notion of the absoluteness of science and the notion of one literal truth. What is important to appreciate in the advance of science, however, is not that the novel idea here or the counterintuitive notion there answers traditional questions, but the fact that virtually all of our traditional categories are being questioned, and this in turn, in a self-referential way, questions the basic assumptions of science. What is occurring is a blurring of traditional methodologies, of traditional compartmentalizations, including the notion that knowledge comes in only one size and one color, and it must be literal.

The contemporary discoveries of science have a great many exciting things to say to us, but we ought to be willing to listen to them fully and to understand their most profound implications. Slawinski has 
retained seventeenth-century assumptions about metaphysical and religious questions, and has merely tried to deal with them through some technological achievements of the twentieth century; but the exciting story that comes out of twentieth-century science is that the categories of the seventeenth century are inadequate. Even the questions themselves may have to be recast, including the notion of survival. At any rate, until we understand how the revolutions in twentieth-century science have changed our categories of understanding, we can at best utilize scientific advances in physics as analogies in helping us to understand possibilities surrounding traditional metaphysical problems, rather than literally answering them. 